Research Article

\title{
Flow Management with Differentiated Classes of Service and Quality of Experience
}

\author{
João Silva (iD, João Ponte, João Pedro Lopes, and Nuno Souto 1 \\ Instituto Universitário de Lisboa (ISCTE-IUL), Instituto de Telecomunicações, Lisbon, Portugal \\ Correspondence should be addressed to João Silva; jcmsa@iscte.pt
}

Received 15 December 2019; Revised 7 February 2020; Accepted 17 February 2020; Published 8 June 2020

Academic Editor: Liansheng Tan

Copyright (C) 2020 João Silva et al. This is an open access article distributed under the Creative Commons Attribution License, which permits unrestricted use, distribution, and reproduction in any medium, provided the original work is properly cited.

\begin{abstract}
Recently, mobile networks have been overloaded with a considerable amount of data traffic. This paper proposes a brokerage service for mobile environments, using policies and quality metrics and ensuring better usage of network resources with more fine-grained management based on flows, and expands previous work (Silva, et al.) with the novel addition of differentiated classes of service. A closed innovative control loop included in a flexible brokerage service in the network, as well as agents at the mobile terminals, detects and counteracts an abnormal data load in any access technology. This allows the terminals to make wellinformed decisions about their connections to improve the number of connected flows per technology and the individual service level offered to each flow. Simulation results indicate that the current proposed management solution optimizes the usage of available $4 \mathrm{G} / 5 \mathrm{G}$ network resources among a high number of flows in several usage scenarios where the technologies are extremely overloaded, while at the same time, protecting the higher classes of service.
\end{abstract}

\section{Introduction}

According to the Cisco Global Mobile Data Traffic Forecast, mobile data traffic will grow at a compound annual growth rate of 47 percent from 2016 to 2021 [1]. It is also expected that global mobile data traffic will increase sevenfold between 2016 and 2021 [1]. Due to the lack of capacity in the mobile network infrastructure, originally dimensioned to support only voice calls and text messages, it is envisioned that the existing macrocells will be increasingly enhanced from a capacity standpoint with a new breed of low-power nodes but from distinct technologies like public space femtocells and WiFi hotspots.

The current paper follows on from previous work [2] that implemented and designed a brokerage service between two different technologies and/or operators. Expanding on the same used simulator, this paper implemented two different classes of service, with one class for protected and priority flows and the other class for normal flows.

As a basis, previous papers modeled and performed an initial analytic study to satisfy as much as possible the apparently incompatible major requirements of both network operators and users [3], a design of a complete solution to control a heterogeneous wireless access network infrastructure in case of an abnormal data load $[4,5]$, and a brokerage service to manage a heterogeneous wireless access network in a very efficient way [6]. Previous work also suggests that a network architecture with an enhanced integration design, in which several network units collaborate in the discovery of relevant network status, can diminish the necessary time for network selection and increase its reliability [7]. The authors built a sophisticated network brokerage scheme simulator in [2], based on the following assumptions:

(i) Individual influence of each flow on the access selection process, instead of solving this issue at the users/terminals level, as proposed in [6]

(ii) Possibility of upgrade/downgrade (UD) of each individual flow rate, depending on the available network resources and load

(iii) Implementation of a channel probabilistic model for dropping flows with a new decision level based on the available network resources 
The work in this paper added differentiated classes of service to the previous simulator, in order to distinguish between priority and nonpriority flows and study its impact on the quality of experience of each user and flow type.

Combining all these new functionalities, the current work studies the overall performance results stemming from having 2 different qualities of service, which for the simulator are dubbed normal and gold users. The gold users represent the highest quality of service and experience, since gold users are protected from downgrades, handovers, and blocks; in fact, this paper aims to quantify the advantages of such performance gains, so that the business part can infer the financial value of such performance advantages over the normal case scenario. Similar work can be found in [8] (optimization of spectrum and radio resource utilization) and [9] (based on a mathematical heuristic), though our approach is simpler (easier to implement), with similar results and with the addition of differentiated classes of service.

The remainder of the paper is organized as follows. Section 2 contextualizes the current work in the literature and highlights the novel aspects of our research. Section 3 discusses the usage scenario of the work. Section 4 describes the design of the management solution. Section 5 evaluates the proposal with the new functional features. Finally, Section 6 discusses main research contributions and future work.

\section{Related Work}

The current work discusses how a brokerage system can manage congestion in a network access environment, balancing high data demand among distinct wireless access technologies and guaranteeing a satisfactory connection quality to mobile flows. The connection quality is measured in terms of the bitrate offered by each wireless link to each flow. In addition, the flow mobility in the current work is mainly necessary to balance the traffic load among the deployed access technologies in a specific location. For distinct scenarios about mobility support, a comprehensive survey is available in [10]. There are some open issues in the integration of distinct wireless access technologies [11], namely, the following: load balancing, traffic management among networks, connection admission, network selection, and resource sharing. In addition, this work supports the dynamic calculation of network attachment point (NAP) quality at the network in terms of the wireless status. Furthermore, the terminal side supports a dynamic cost function to rank target NAPs based on the connection quality offered by each one of these. In this way, the final decision in terms of NAP selection per flow is issued at terminals and supported by the distributed broker units at the network infrastructure. A literature review about network brokers is available in [6]. This work proposes a novel bandwidth broker that uses policies for managing a high amount of unicast data load crossing a heterogeneous NGN environment and enhancing some traditional network services, like flow admission and network selection. In fact, Figure 1 shows a complete heterogeneous wireless access infrastructure that is controlled at the network edge by broker units with distinct management roles. Broker units control one or more access technologies like WiMAX and cellular technologies, through a peering communication protocol.

The current broker architecture has an innovative hybrid design, aggregating both centralized and distributed functionalities in comparison with [12]. In addition, the current proposal is more flexible than [12] because the former can operate in both interdomain and intradomain networking scenarios. The current proposal also extends previous work [13], which only manages an $802.11 \mathrm{~b}$ access network. In fact, the current proposal can control a network infrastructure, composed by any access technology, like [14]. Nevertheless, according to our knowledge, a major difference from [14] is that our work supports as a novelty the downgrade/upgrade of each individual flow according to the network available resources and load demand (present in [2]), along with differentiating between different classes of service, which is the main contribution of this paper.

\section{Scenario and Requirements}

This section discusses the scenario, the addressed problems, and the functional requirements for a heterogeneous network infrastructure. Figure 1 illustrates the scenario of this work, where there are areas covered by several wireless access networks, with each one possibly administered by a distinct network provider.

The load may vary considerably during the day not only in terms of the number of flows requiring connection, but also in terms of requirements (e.g., audio/video and high/ low bitrate). Available resources of each access network may not be enough to support a good connection quality for the high number of flows, due to either poor wireless coverage or insufficient network capacity.

The current paper and simulations are built on top of the work developed in [2], which proposed a cooperative model among network providers, coordinated by a brokerage service on the edge of the network, to enhance the service of wireless connectivity provided by a heterogeneous network infrastructure.

As shown in Figure 1, the brokerage functionality is completely distributed among Points of Presence (PoPs) and access networks. There are several broker units located in convenient locations in the wired infrastructure. A PoP is a location where several wireless access technologies exchange their traffic with the Internet through backhaul links. In this scenario, it is assumed that each access technology has a well-provisioned backhaul, and the broker can optionally offer an additional backhaul to expand the connection capacity of any access technology. The broker enables a flexible service to efficiently manage the edge of the network infrastructure on behalf of the network providers. In addition, this local brokerage service can coordinate its management decisions with remote brokers located at neighbor PoPs in a peer-to-peer communication (link 6 of Figure 1). In this way, the last coordination is very useful to anticipate congestion 


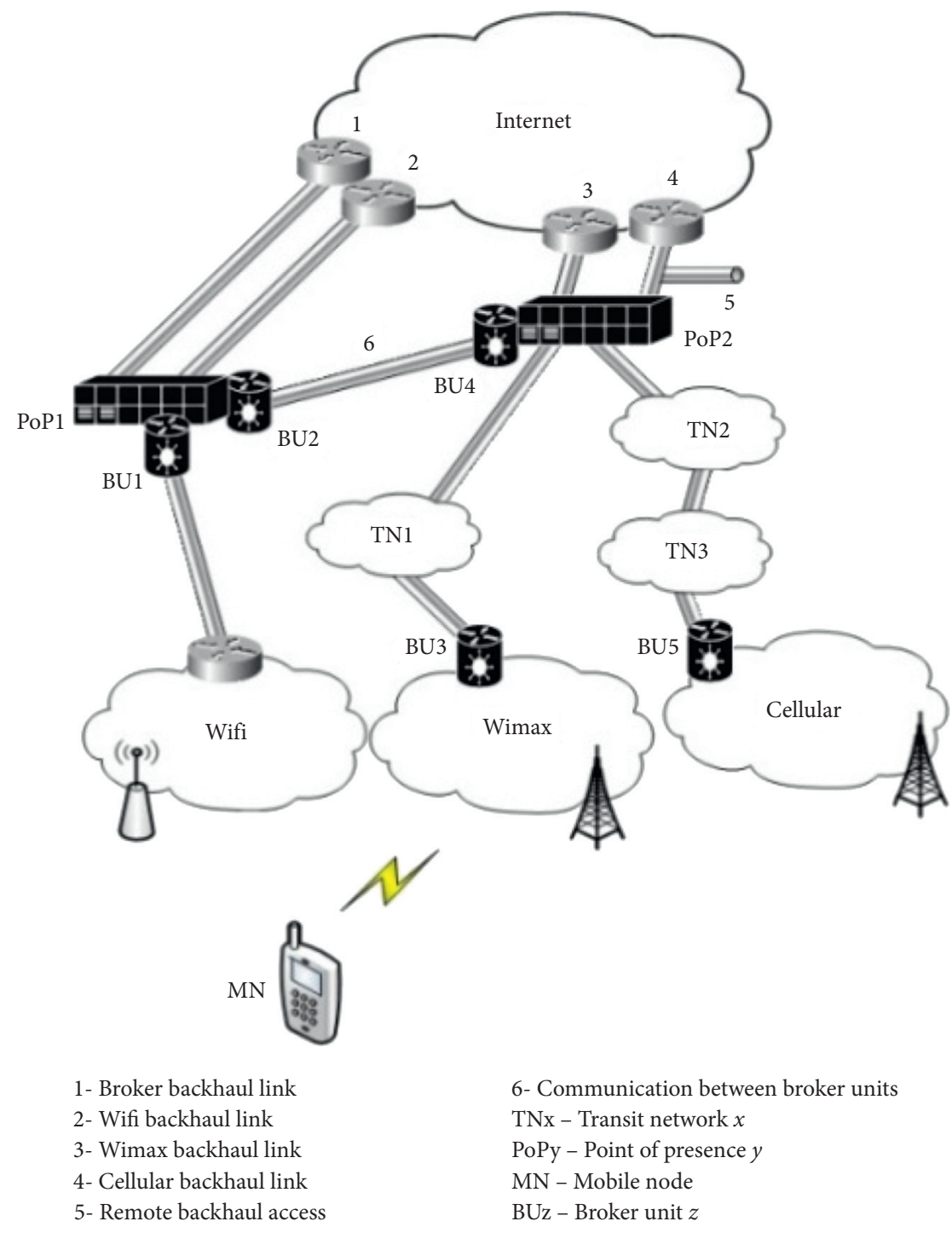

FIGURE 1: Scenario of the heterogeneous network.

in certain areas using information about load movements from neighbor areas towards the former area.

To guarantee that the network infrastructure operates correctly, several requirements/assumptions should be verified by a management proposal based on a brokerage service. First, each customer obtains a connection to the network, paying a fee that depends on the subscription contract with his network provider. This fee will depend on the types and the classes of service required for each type (main novelty of this paper). In addition, the roaming cost is shared (or paid) among the providers, transparently to the roaming users.

Second, the brokerage service evaluates the connection quality associated with each NAP, after which the brokerage service detects any congested links and remediates a congestion situation applying adequate policies to the network equipment, using a reactive mode.

Finally, an additional predictive mode can be considered for the brokerage service. This last operation mode could evaluate the success/failure degree of each applied policy, produce a ranked list of policies, learn how a network problem occurred and has been solved, and predict when the next problem could happen.

An example of a real scenario like the one visualized in Figure 1 is explained as follows. Here, for a network connection for a certain flow, the passengers of a train can choose between a WiFi AP located inside the train and external LTE macrocells covering the train route. Depending on several aspects, like the flow requirements, remaining energy in the terminal, the load of each technology, the contracted service level, the train location, and the train speed, the most suitable NAP that each flow should connect to can change during the train journey. Consequently, the brokerage service can balance the high load among the available connection capacities offered by the complete set of available NAPs, from the two distinct access technologies.

The broker units can be deployed in a very simple way as a software routine at already existing network units like either the access router of $\mathrm{WiFi}$ or the ASN gateway of WiMAX. In addition, multimedia independent handover 
services (i.e., IEEE 802.21) can be used to enable the brokerage service to supervise and control several access technologies, either locally at a single network node or remotely between distinct network nodes [7].

\section{Solution Design}

This section presents the design of a brokerage service, using a set of decision thresholds based on policies, to efficiently manage the available network resources of a heterogeneous infrastructure. Figure 2 shows the decision thresholds for a wireless access technology: admission of new flows, management of accepted flows according to the network status, and flows dropped by the influence of the physical channel. Finally, each access technology has a maximum value of connection rate designated by Max_rate, and the previous threshold values are relative to this maximum. The brokerage service is the same as that used in [2], running continuously in consecutive iterations, with each iteration representing a time period (seconds, in the simulations). Due to the simulation of rush-hour traffic, with an excessive amount of flows logging in at the same time, an iterative model was chosen, where a single iteration of the current model deals with a specific number of new flows, blocks, drops, and terminations of flows. In addition, the next sections discuss the following functional aspects: network selection and flow admission (IV. A), drop of flows in channels (IV. B), and management of connected flows (IV. C).

4.1. Network Selection and Flow Admission. This section discusses the network selection and flow admission features of the brokerage system. The flowchart of these features is visualized in Figure 3. Notice that the algorithm can block some flows when the network load is higher than the value overload and that, when the broker is disabled, the terminal randomly selects an access network (normally, the one offering the best signal to noise ratio (SNR)). When the broker is enabled, the network selection and admission control favor the least constraint network (two settings were tested: (a) two similar networks and (b) two networks where the second network had twice the amount of capacity of the first, $\left.T_{2} / T_{1}=2\right)$.

Another important aspect that was implemented in this paper is the class of service, which in this work is divided between "normal" and "gold" users, whereas in [2] we had the same service level for all users. In this paper, gold users are defined by all users that pay a higher fee than normal users in order to get access to the following perks:

(i) Their flows are always the last ones to be blocked or dropped

(ii) Gold users are always the last ones to be handed over to another network

(iii) Gold users are never downgraded regardless of network condition, which means that they are never upgraded as well
4.2. Flow Drops. This section discusses in what situations the flows are dropped per channel. In fact, some flows are randomly dropped, as was done in [2]. This models the situations where, due to problems occurring in a very busy communication channel (e.g., interference), the flow connections through that channel are abruptly interrupted. In this way, there are three distinct probabilities for dropping a flow, enhancing previous work that had only two drop probabilities [6]. Each drop probability is associated with a distinct level of network load (see Figure 2). A distinct drop probability per technology is also assumed for the same load level, depending on the priority parameter $T_{2} / T_{1}$.

The drop probability depends on the access technology and the load of each technology. In this way, the channel drops flows per technology with a probability of $0.01 \%$ if the load of that technology is below the quality threshold (QT) associated with it. Otherwise, if the load is inside the range [QT, overload], then the channel drops flows with a probability of $0.02 \%$. Alternatively, the channel drops more flows with a probability of $0.5 \%$ because the wireless access technology is more overloaded. The chosen values for the channel drops were estimated from BER (bit error rate) performances for OFDM schemes, with a special focus on LTE-A $[15,16]$ with the Indoor-A channel model [17]. Note that the flow drop probability is transversal to all classes of service, and thus the higher classes of services must have preventive measures to minimize their drop probabilities, namely, in downgrading of performing handovers of "normal" flows.

Additionally, there is a more deliberate way of dropping flows (see Figure 4). If the current technology load is higher than its maximum rate, an excess rate is computed (this only happens when there is handover delay; see ahead). If it is higher than 0 , normal user's flows are dropped starting from the highest data service ones to the lowest. If the excess rate is still not 0 or under after dropping normal user's flows, the broker will start dropping gold user's flows-this is a last resort scenario and the only case where gold users are penalized by the system - and the system will seek to minimize the impact, dropping the gold users with high bitrates first, starting with the one that was connected the longest.

4.3. Management of Connected Flows. This section explains the rate management of already connected flows to reduce the number of blocked flows. The flows are managed with policies that depend on the channel overload. The downgrade and upgrade routines are described in [2]. Figure 5 portrays the flowchart that describes how a handover is performed when one technology is experiencing too much load. Before performing a handover, the broker can apply a delay to it to emulate what can happen in a real network. To achieve this, the broker takes a screenshot of the current state of the network and performs the handover a few seconds later, which could mean that the flow will be transferred to a network that got fully loaded in the meantime. This can have nefarious implications (e.g., the downgrade of another user's flow or the drop of a user altogether). Afterwards, if a technology load is in the $[0, \mathrm{QT} *$ Hyst $]$ range and there is a 


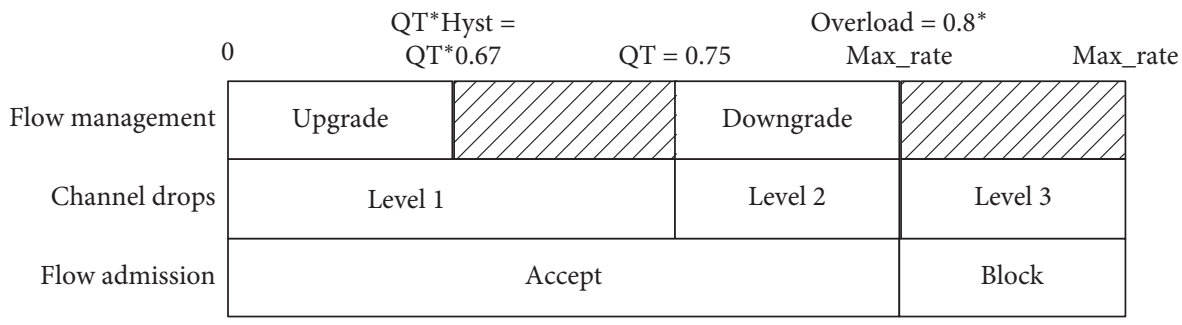

FIgURE 2: Decision thresholds for the brokerage service.

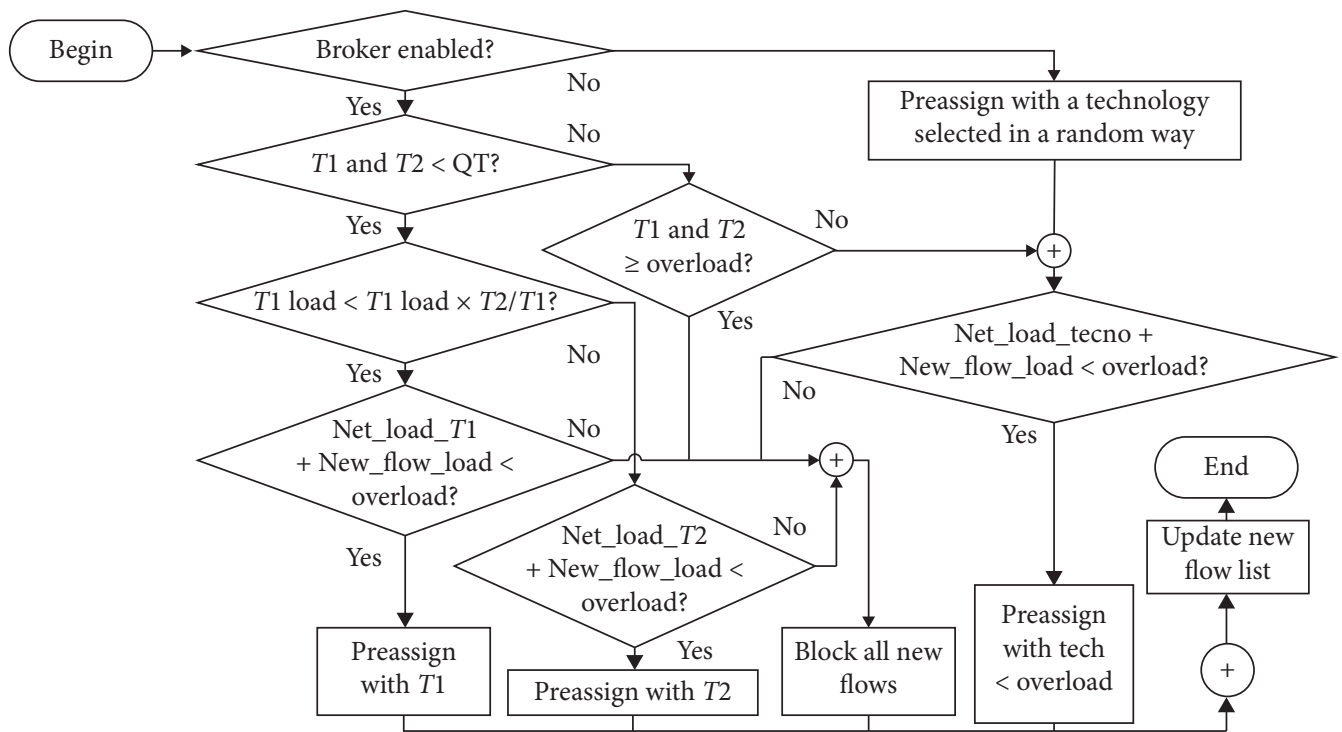

FIGURE 3: Network selection and flow admission.

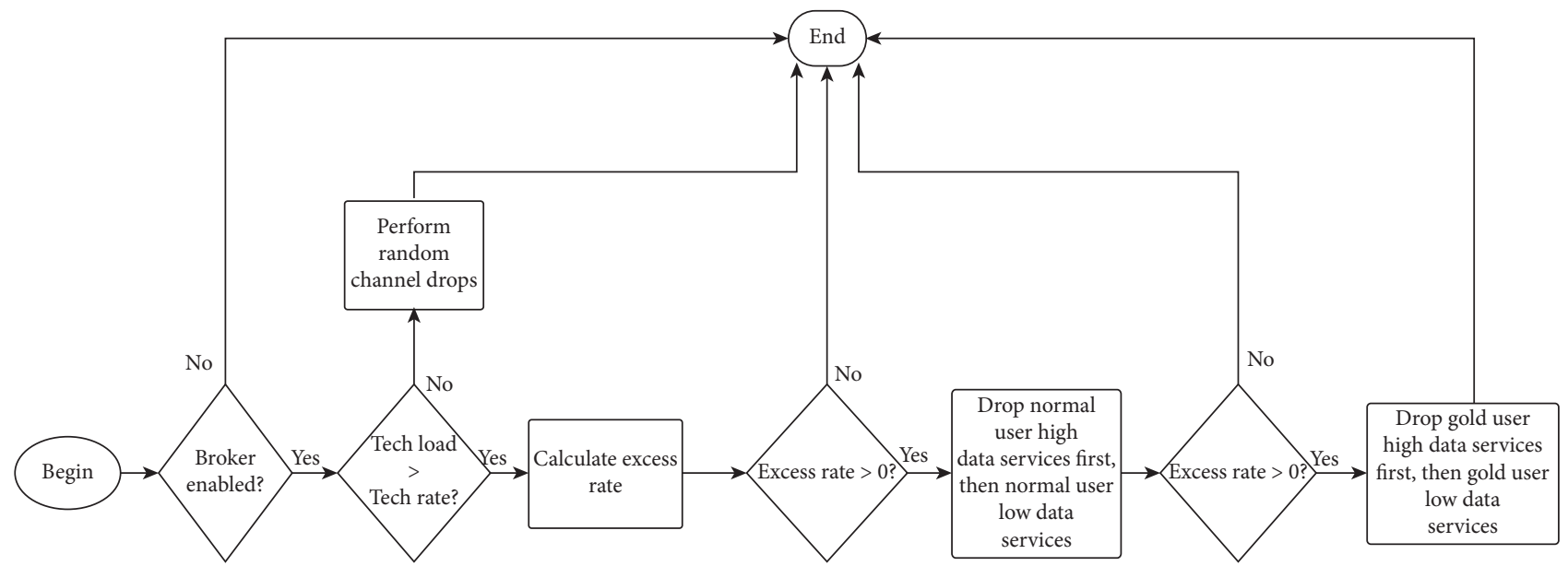

FIgURE 4: Flow drop flowchart.

technology that is in the [QT, Max_rate] range, a handover is performed to the former. Normal and gold users with high data services are transferred first, followed by normal and gold users with low data services. In both instances, gold users are always transferred last.

\section{Evaluation}

This section evaluates the brokerage service when it manages the available connection capacity of a heterogeneous network infrastructure, ensuring the best possible connection 


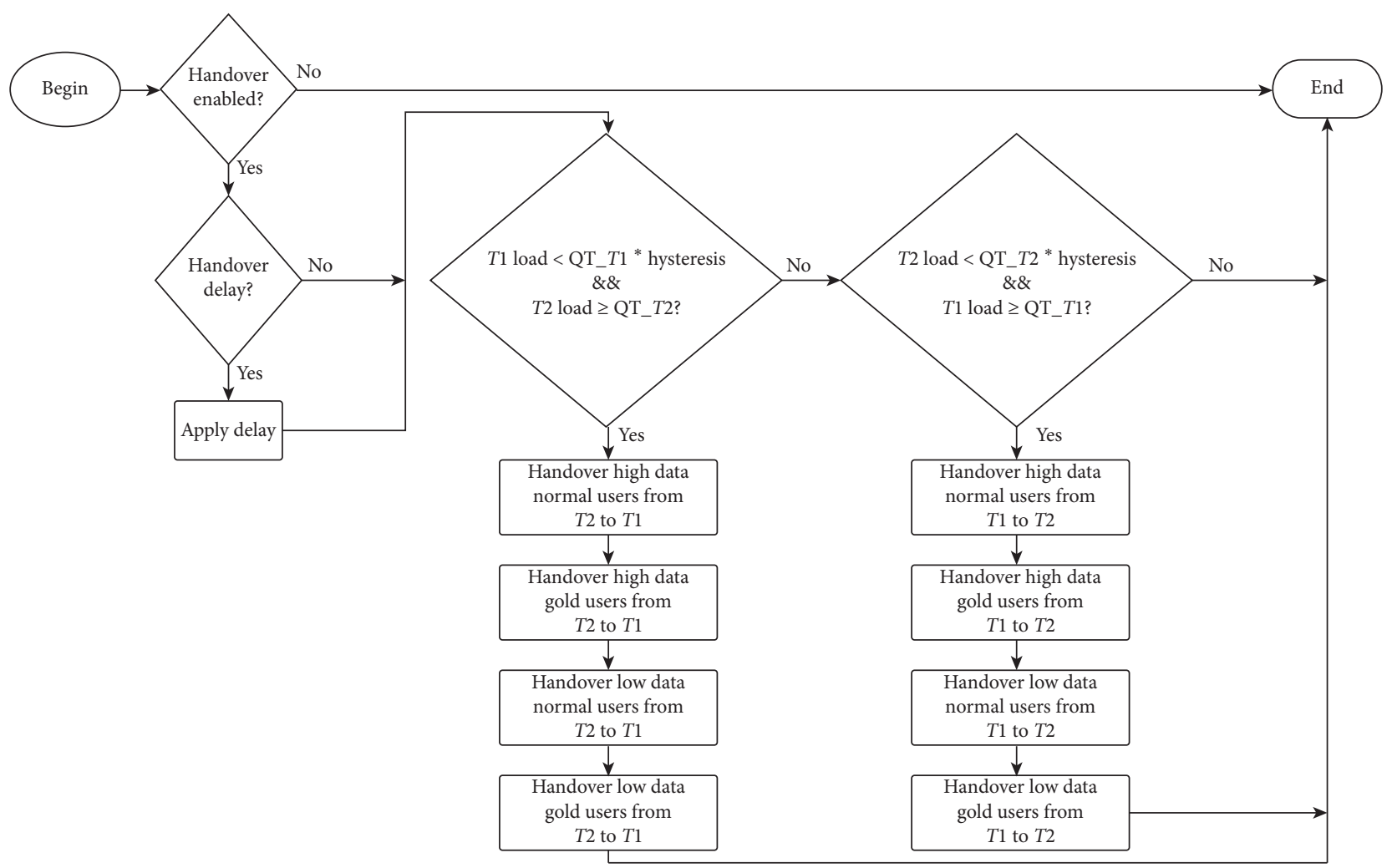

FIgURE 5: Flow handover flowchart.

quality to each flow in scenarios where the wireless access technologies are severely overloaded. The scenario of Figure 1 was used, with 2 access technologies from two different providers: $T_{1}$ (LTE-A) and $T_{2}$ (WLAN-WiFi). Notice, however, that the results portrayed in this paper can be used either for similar technologies from different providers, different technologies from the same provider, or different technologies from different providers. Since nowadays the interworking among 5G, LTE, and non-3GPP is issued, the proposed brokerage service can be used mainly for separating providers, although it can also serve the purpose of separating noninterworking technologies.

The Indoor-A channel model was used, and an overloaded setting simulated (the initial linear climb in Figures 6-7 representing the system loading from 0 users to full saturation, with 10 new users every second wanting to join). At the end of the simulation, from instance $1000 \mathrm{~s}$ onwards, no new users will join the network, in order to see the system recover and deal with all users until all end their call.

From the analysis of the mean load history of gold and nongold users, it is clear that gold users have a higher throughput value throughout the duration of the simulation, even with an increased ratio of gold to nongold users. This is achieved at the expense of nongold users' throughput as shown by the drop in nongold user throughput in Figure 7 when compared to Figure 6 . This effectively means that, in a normally functioning network, the broker algorithm guarantees that gold users will always have more available bandwidth than their nongold counterparts.

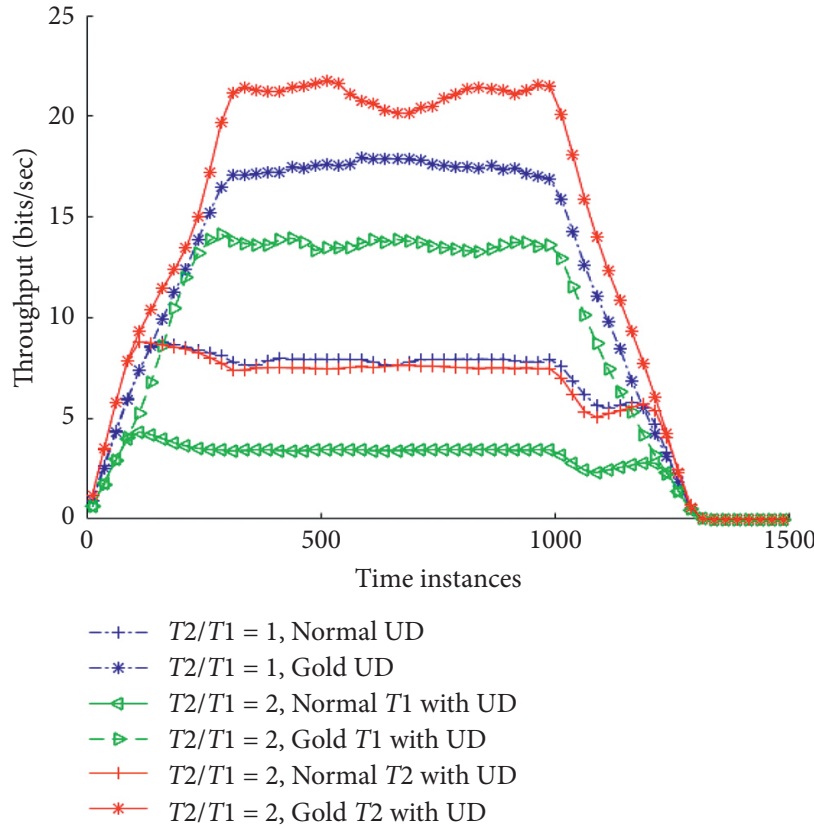

Figure 6: Mean load history of all users with $10 \%$ gold users.

Figures 8-10 present simulated average user satisfaction on a logarithmic scale. Satisfaction was defined as follows: a user that is not subject to any downgrade/handover/block or drop has a satisfaction of $100 \%$; a dropped call will have a satisfaction of $10 \%$ and a blocked call $70 \%$. A handover will have a multiplying factor effect of $80 \%$, a downgrade will 


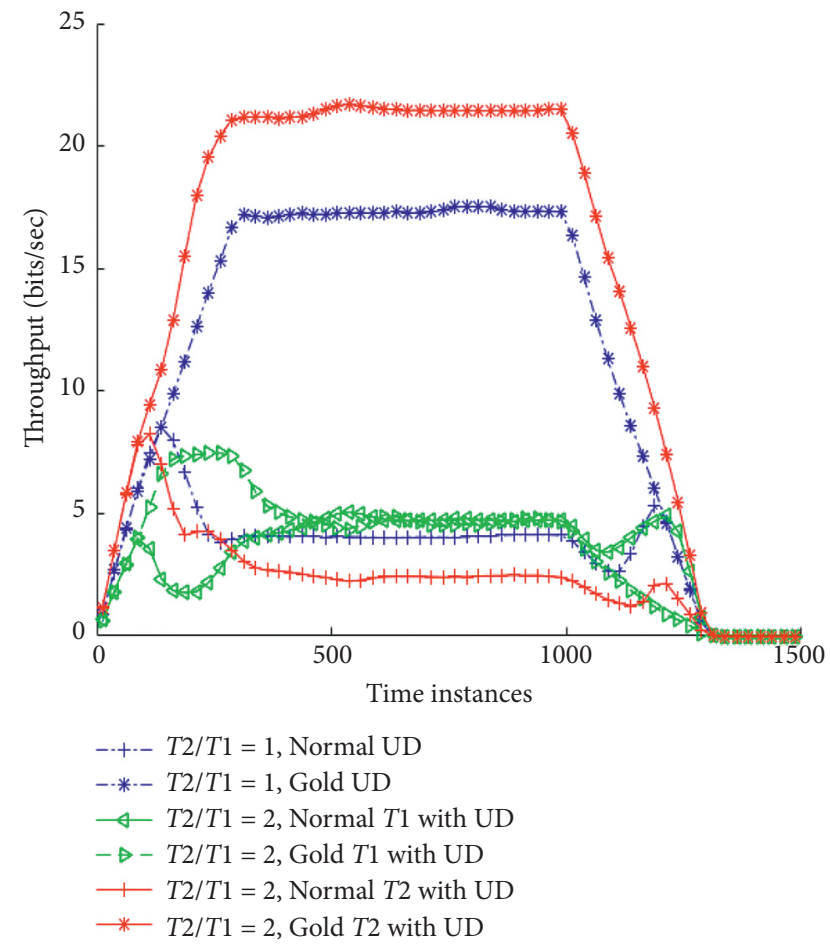

FIgURE 7: Mean load history of all users with 50\% gold users.

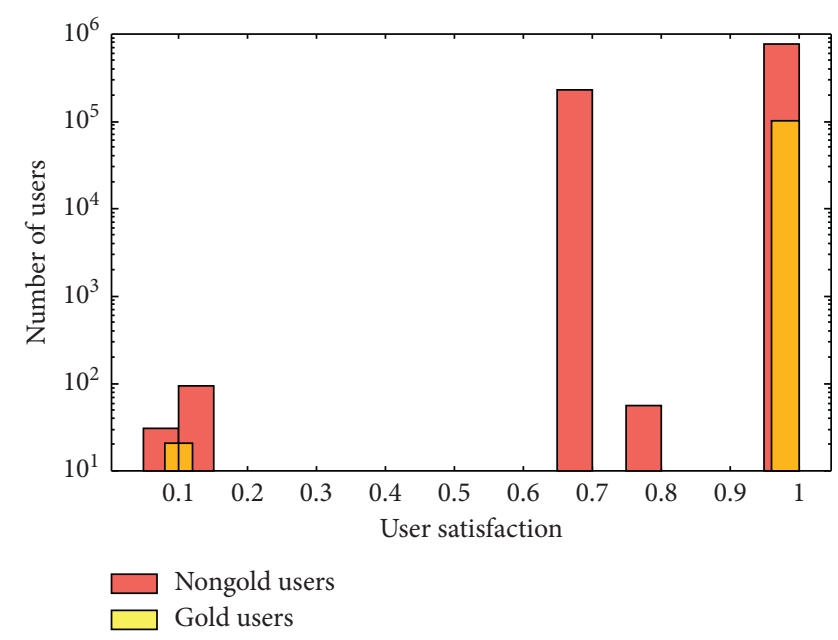

Figure 8: Simulated average customer satisfaction with $10 \%$ gold users.

have a multiplying factor of $85 \%$, and an upgrade will have a factor of 1.1 (there will only be an upgrade if a downgrade was applied before). Notice that if a call is both downgraded and upgraded, then the resulting satisfaction will be (if no handover was performed) of $1 \times 0.85 \times 1.1=0.935$.

Due to the broker algorithm, most gold users will have near $100 \%$ satisfaction with the exceptions of those users whose calls were randomly dropped (in these cases, their satisfaction drops to $10 \%)$. As for nongold users, since they can have their call blocked or downgraded, their average satisfaction fluctuates but mostly remains around $70 \%$ or

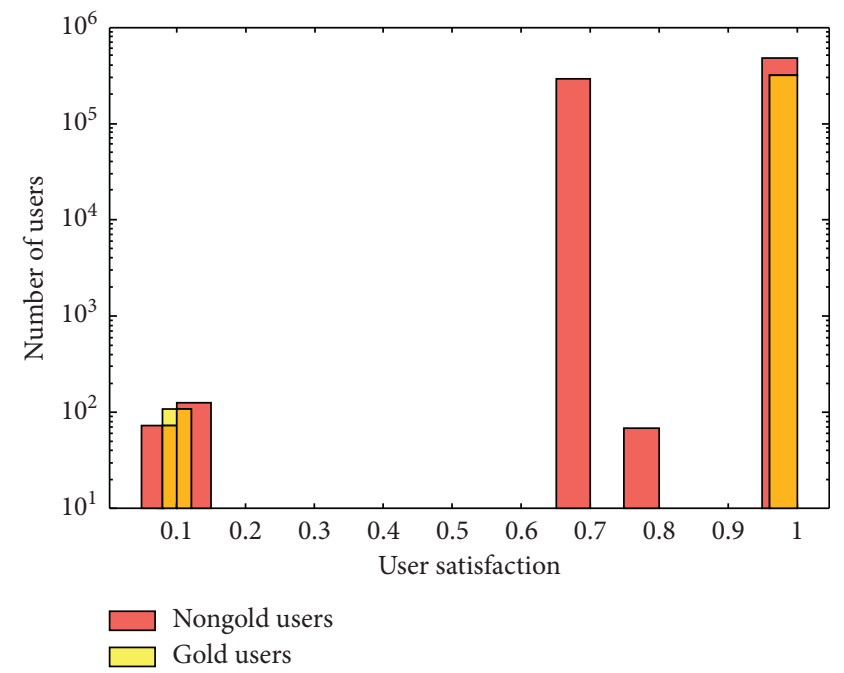

FIGURE 9: Simulated average customer satisfaction with $30 \%$ gold users.

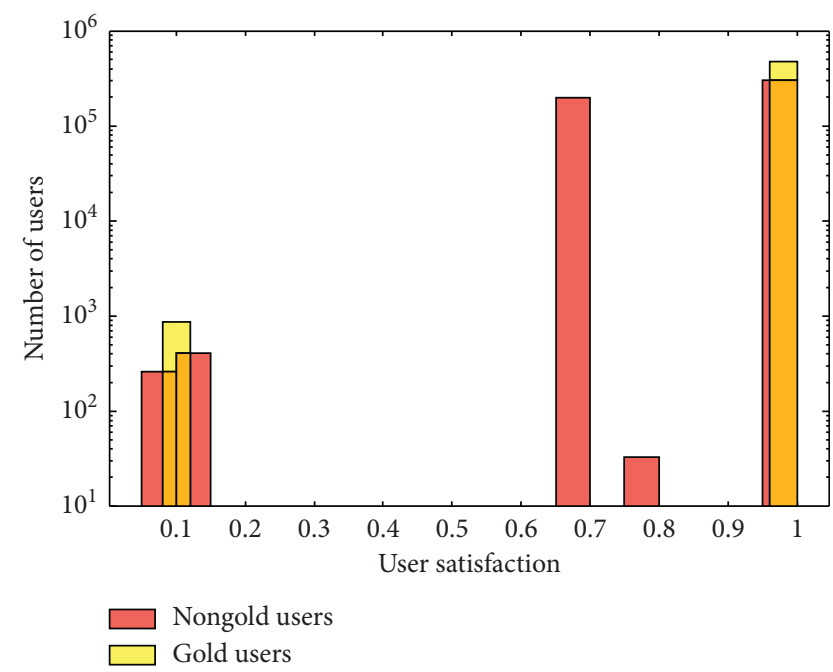

FIGURE 10: Simulated average customer satisfaction with 50\% gold users.

$100 \%$ satisfaction. The results reveal that the average satisfaction will be higher for gold users, as expected, since their perception of the quality of service will greatly exceed that of the nongold users, even with an even 50/50 split of user types.

When comparing results to [2], we can see that the protected flows are privileged to having nearly $100 \%$ satisfaction levels, as long as the protected flows ratio to other flows is kept less than 50\%. Normal users are subjected to downgrades and handovers, and thus their satisfaction levels would be around $70 \%-80 \%$.

We can also conclude that protection only comes into play on occasions of load saturation, which happens in about $5 \%$ of the times during the day, which means that commercially only very specific clients would be willing to pay 
the premium for protection; thus, a scenario with $10 \%$ gold users or less would be the most realistic setting.

\section{Conclusions}

The current work expands on the advantages of a new functionality to better manage flows in a distributed way, by means of a brokerage service in a heterogeneous wireless access infrastructure, by using differentiated quality of service. With this work, we were able to portray the advantages of paying for a higher quality of service even in highly congested scenarios with the broker algorithm still managing to maintain acceptable quality of service for the regular users, especially considering that the scenarios described in the present work are representative of extreme congestion which contrasts with the much lower regular network usage.

From the results, we can see that indeed it is possible to have two (or more) differentiated classes of services, with the broker rearranging the normal users as best as possible before moving on to the gold users. In fact, we can see that, in an overloaded scenario, the gold users are highly protected and thus mostly end up with $100 \%$ satisfaction level (the ones that were not subject to random channel drops, which the broker did not control). Only under very specific conditions would the broker start downgrading gold users (scenarios with a very high percentage of gold users, which were not simulated since gold users should represent about $20 \%$ of the market segment). Future work will address scenarios where flow management ensures energy efficiency at the terminal.

\section{Data Availability}

All data were gathered from public references that are cited in the article.

\section{Conflicts of Interest}

The authors declare that they have no conflicts of interest.

\section{Acknowledgments}

This work was funded by FCT/MCTES through national funds and when applicable, cofunded by EU funds under the project UIDB/EEA/50008/2020.

\section{References}

[1] Cisco Visual Networking Index, "Global mobile data traffic forecast update," 2016, https://www.cisco.com/c/en/us/solutions/ collateral/service-provider/visual-networking-index-vni/mobilewhite-paper-c11-520862.pdf (verified in 2018/09/26).

[2] J. C. Silva, J. A. Moura, R. N. Marinheiro, and J. M. D. A. Almeida, "Optimizing 4G networks with flow management using an hybrid broker," in Proceedings of the Springer International Conference on Advances in Information Technology and Mobile Communication-AIM, Elsevier, Bangalore, India, pp. 290-298, April 2013.

[3] J. Moura, M. Dunmore, and C. Edwards, "Next generation network management of heterogeneous mobile users," in
Proceedings of the 6th ACM International Symposium on Mobility Management and Wireless Access, ACM, New York, NY, USA, pp. 111-118, 2008.

[4] J. Moura, M. Dunmore, and C. Edwards, "Next generation network management of heterogeneous mobile users," Journal of Network and Systems Management, vol. 2008, pp. 111-118, 2008.

[5] J. Moura, M. Dunmore, and C. Edwards, NGN Model with Heterogeneous Mobile Customers, Trilogy Future Internet Summer School, Padua, Italy, 2009.

[6] J. Moura, J. Silva, and R. N. Marinheiro, "A brokerage system for enhancing wireless access," in Proceedings of the International Conference on Communications and Signal Processing (MIC-CSP2012), Chemnitz, Germany, March 2012.

[7] R. Fänge and U. Lidman, "Secretion of sulfuric acid in Cassidaria echinophora Lamarck (Mollusca: mesogastropoda, marine carnivorous snail)," Comparative Biochemistry and Physiology, vol. 53, pp. 101-103, 1976.

[8] A. Galani, K. Tsagkaris, and P. Demestichas, "Information flow for optimized management of spectrum and radio resources in cognitive B3G wireless networks," Journal of Network and Systems Management, vol. 18, no. 2, pp. 125-149, 2010.

[9] T. De Schepper, S. Latré, and J. Famaey, "Scalable load balancing and flow management in dynamic heterogeneous wireless networks," Journal of Network and Systems Managenent, 2019.

[10] X. Yan, Y. Ahmet Şekercioğlu, and S. Narayanan, “A survey of vertical handover decision algorithms in Fourth Generation heterogeneous wireless networks," Computer Networks, vol. 54, no. 11, pp. 1848-1863, 2010.

[11] E. Stevens-Navarro, "Vertical handover in beyond third generation (B3G) wireless networks," International Journal of Future Generation Communication and Networking, vol. 1, no. 1, pp. 51-58, 2008.

[12] C. Bouras and D. Primpas, "Architectures and performance evaluation of bandwidth brokers," International Journal of Network Management, vol. 19, no. 2, pp. 101-117, 2009.

[13] N. Blefari-Melazzi, "Toward an autonomic control of wireless access networks," in Proceedings of the IEEE Globecom, IEEE, Taipei, Taiwan, pp. 954-959, December 2005.

[14] M. Almeida, D. Corujo, S. Sargento, V. Jesus, and R. Aguiar, "An end-to-end qos framework for $4 \mathrm{~g}$ mobile heterogeneous environments," in Proceedings of the OpenNet Workshop, IGI Global, Brussels, Belgium, March 2007.

[15] A. Correia, "Mutiresolution with hierarchical modulations for long term evolution of UMTS," EURASIP Journal on Wireless Communications and Networking, vol. 2009, Article ID 240140, 11 pages, 2009.

[16] R. Dinis, "On the design of turbo equalizers for SC-fde schemes with different error protections," in Proceedings of the IEEE Vehicular Networking Conference, IEEE, Jersey City, NJ, USA, December 2010.

[17] S. K. Yong, R. Banerjae, and H. Zhang, Indoor Channel Model for TGah, IEEE 11-11-0361-00-00ah, 2011. 